\title{
VARIATIONS ON A THEOREM OF DAVENPORT CONCERNING ABUNDANT NUMBERS
}

\author{
EMILY JENNINGS, PAUL POLLACK ${ }^{凶}$ and LOLA THOMPSON
}

(Received 6 June 2013; accepted 2 July 2013; first published online 12 September 2013)

\section{Abstract}

Let $\sigma(n)=\sum_{d \mid n} d$ be the usual sum-of-divisors function. In 1933, Davenport showed that $n / \sigma(n)$ possesses a continuous distribution function. In other words, the limit $D(u):=\lim _{x \rightarrow \infty}(1 / x) \sum_{n \leq x, n / \sigma(n) \leq u} 1$ exists for all $u \in[0,1]$ and varies continuously with $u$. We study the behaviour of the sums $\sum_{n \leq x, n / \sigma(n) \leq u} f(n)$ for certain complex-valued multiplicative functions $f$. Our results cover many of the more frequently encountered functions, including $\varphi(n), \tau(n)$ and $\mu(n)$. They also apply to the representation function for sums of two squares, yielding the following analogue of Davenport's result: for all $u \in[0,1]$, the limit

$$
\tilde{D}(u):=\lim _{R \rightarrow \infty} \frac{1}{\pi R} \#\left\{(x, y) \in \mathbb{Z}^{2}: 0<x^{2}+y^{2} \leq R \text { and } \frac{x^{2}+y^{2}}{\sigma\left(x^{2}+y^{2}\right)} \leq u\right\}
$$

exists, and $\tilde{D}(u)$ is both continuous and strictly increasing on $[0,1]$.

2010 Mathematics subject classification: primary 11N60; secondary 11A25, 11N37.

Keywords and phrases: abundant number, distribution function, mean values of multiplicative functions, sum-of-divisors function.

\section{Introduction}

Recall that a natural number $n$ is said to be abundant if $\sigma(n)>2 n$, where $\sigma(n):=\sum_{d \mid n} d$ denotes the usual sum-of-divisors function. Answering a question of Bessel-Hagen, Davenport [2] showed that the set of abundant numbers possesses an asymptotic density. In fact, he proved the more precise result that $n / \sigma(n)$ possesses a continuous distribution function. In other words, the limit

$$
D(u):=\lim _{x \rightarrow \infty} \frac{1}{x} \sum_{\substack{n \leq x \\ n / \sigma(n) \leq u}} 1
$$

exists for all $u \in[0,1]$ and varies continuously with $u$. We have followed modern conventions in writing the condition on $n / \sigma(n)$ as a nonstrict inequality, but since $D(u)$ is continuous, whether or not we allow $n / \sigma(n)=u$ does not change the value of $D(u)$. Recent work of Kobayashi [8] shows that $0.24761<D\left(\frac{1}{2}\right)<0.24765$, so that just under one in four numbers are abundant. 
The purpose of this paper is to establish analogues of Davenport's theorem where the uninteresting summand 1 appearing in (1.1) is replaced with $f(n)$ for certain complex-valued multiplicative functions $f$. We prove two theorems in this direction, the first of which is as follows. Recall that an arithmetic function $f$ is said to possess a mean value if $(1 / x) \sum_{n \leq x} f(n)$ approaches a (complex number) limit as $x \rightarrow \infty$.

THEOREM 1.1. Let $f$ be a multiplicative function that is bounded in mean square, that is,

$$
\limsup _{x \rightarrow \infty} \frac{1}{x} \sum_{n \leq x}|f(n)|^{2}<\infty .
$$

Suppose that for every nonnegative integer $k$, the function $n \mapsto f(n)(n / \sigma(n))^{k}$ possesses a mean value. Then for every real $u \in[0,1]$, the limit

$$
D_{f}(u):=\lim _{x \rightarrow \infty} \frac{1}{x} \sum_{\substack{n \leq x \\ n / \sigma(n) \leq u}} f(n)
$$

exists. Moreover, $D_{f}(u)$ is continuous as a function of $u$.

Theorem 1.1 is proved in Section 2. In the same section, we obtain the following consequences. From now on, let $p$ be a prime variable.

Corollary 1.2. Let $f$ be a multiplicative function bounded in mean square. Then the hypotheses of Theorem 1.1, and hence also its conclusion, hold if

$$
\sum_{p} \frac{|f(p)-1|}{p}<\infty \quad \text { and } \quad \sum_{p} \sum_{j \geq 2} \frac{\left|f\left(p^{j}\right)\right|}{p^{j}}<\infty .
$$

If $|f(n)| \leq 1$ for all $n \in \mathbb{N}$, then (1.3) can be replaced with the weaker assumption that the series

$$
\sum_{p} \frac{f(p)-1}{p}
$$

converges (possibly conditionally).

Corollary 1.3. Let $f$ be a multiplicative function with $|f(n)| \leq 1$ for all natural numbers $n$. Suppose that $f$ has mean value zero. Suppose further that there is no real number $\beta$ with the property that $f\left(2^{j}\right)=-2^{i j \beta}$ for every positive integer $j$. Then the function $D_{f}(u)$ defined in (1.2) vanishes identically for all $u \in[0,1]$.

EXAMPLES 1.4.

(i) A simple example of a function satisfying the hypotheses of Corollary 1.2 is the indicator function of the squarefree numbers (or more generally, the $\ell$-free numbers). The hypotheses of that result also hold for the functions $(\varphi(n) / n)^{z}$ and $(\sigma(n) / n)^{z}$, for any complex number $z$. To obtain a result for $\varphi(n)$ or $\sigma(n)$, one can 
apply Corollary 1.2 to $\varphi(n) / n$ or $\sigma(n) / n$, and then remove the weight of $1 / n$ by partial summation. Indeed, whenever the conclusion of Theorem 1.1 holds,

$$
\lim _{x \rightarrow \infty} \frac{1}{x^{2}} \sum_{\substack{n \leq x \\ n / \sigma(n) \leq u}} n f(n)=\frac{1}{2} D_{f}(u) .
$$

(ii) A natural family of examples satisfying the hypotheses of Corollary 1.3 are the functions $\lambda_{a, q}(n):=\exp (2 \pi i(a / q) \Omega(n))$ with $q$ not dividing $a$. Here, as usual, $\Omega(n)$ denotes the number of prime factors of $n$ counted with multiplicity. That all of the functions $\lambda_{a, q}(n)$ have mean value zero seems to have been first proved by Pillai and Chowla [10] (alternatively, this assertion follows from a beautiful theorem of Halász, quoted in Section 2). The conclusion of Corollary 1.3 for this family leads, via the orthogonality relations for additive characters, to the following pretty consequence.

Fix $q \in \mathbb{N}$ and fix $0<u \leq 1$. As $n$ ranges over the solutions to $n / \sigma(n) \leq u$, the values $\Omega(n)$ are equidistributed modulo $q$.

The nontrivial Dirichlet characters form another natural class of examples. Here the corresponding conclusion is:

Fix $q \in \mathbb{N}$ and fix $0<u \leq 1$. The solutions $n$ to $n / \sigma(n) \leq u$ that are relatively prime to $q$ are equidistributed among the coprime residue classes modulo q.

Actually, for this deduction to be valid, one must know that a positive proportion of solutions to $n / \sigma(n) \leq u$ are coprime to $q$. This will follow from Theorem 1.5 below. (As pointed out to the second author by Greg Martin, this equidistribution result can also be obtained by the method of moments.)

For our second theorem, we restrict attention to nonnegative functions $f$ (assumed not to vanish identically). While Theorem 1.1 applies perfectly well to many nonnegative $f$, for others it is simply not the right tool for the job. An illustrative example is provided by the divisor function $\tau$. The mean value of $\tau$ on the interval $[1, x]$ is asymptotic to $\log x$, as $x \rightarrow \infty$. Thus, to obtain the 'correct' analogue of Davenport's theorem, we should not be dividing by $x$ in (1.2) but rather by something proportional to $x \log x$. More generally, for a nonnegative function $f$, we ought to normalise by the factor

$$
S(f ; x):=\sum_{n \leq x} f(n) .
$$

We are thus led to define

$$
\tilde{D}_{f}(u)=\lim _{x \rightarrow \infty} \frac{1}{S(f ; x)} \sum_{\substack{n \leq x \\ n / \sigma(n) \leq u}} f(n),
$$

whenever the limit exists. We can now state our second main result. 
THEOREM 1.5. Suppose that $f$ is a nonnegative multiplicative function with the property that as $x \rightarrow \infty$,

$$
\sum_{p \leq x} f(p) \frac{\log p}{p} \sim \kappa \log x
$$

for some $\kappa>0$. Suppose also that $f(p)$ is bounded for primes $p$ and that

$$
\sum_{p} \sum_{j \geq 2} \frac{f\left(p^{j}\right)}{p^{j}}<\infty
$$

If $\kappa \leq 1$, suppose further that

$$
\sum_{p^{j} \leq x} f\left(p^{j}\right) \ll_{f} \frac{x}{\log x} \quad(\text { for } x \geq 2) .
$$

Then $\tilde{D}_{f}(u)$ exists for all $u \in[0,1]$ and is both continuous and strictly increasing.

EXAMPLES 1.6.

(i) When $f=\tau$, the hypotheses of Theorem 1.5 hold with $\kappa=2$.

(ii) Let $r(n)=\frac{1}{4} \#\left\{(x, y) \in \mathbb{Z}^{2}: x^{2}+y^{2}=n\right\}$. This function fails the hypotheses of Theorem 1.1 (by not being bounded in mean square), but it satisfies the hypotheses of Theorem 1.5 with $\kappa=1$. Since $\sum_{n \leq x} r(n) \sim(\pi / 4) x$ by simple geometric considerations (see [7, Theorem 339, page 357]), we see that

$$
\tilde{D}_{r}(u)=\lim _{R \rightarrow \infty} \frac{1}{\pi R} \#\left\{(x, y) \in \mathbb{Z}^{2}: 0<x^{2}+y^{2} \leq R \text { and } \frac{x^{2}+y^{2}}{\sigma\left(x^{2}+y^{2}\right)} \leq u\right\} .
$$

The existence and continuity of $\tilde{D}_{r}(u)$ may be thought of as a sum-of-two-squares analogue of Davenport's result.

(iii) Multiplicative sets provide a rich source of examples. Here a set $\mathcal{S}$ of natural numbers is called multiplicative if its indicator function $\mathbf{1}_{\mathcal{S}}$ is multiplicative (see, for instance, [9]). Suppose that $\mathcal{S}$ is multiplicative and contains a well-defined, positive proportion of the primes, in the sense that (1.5) holds with $f=\mathbf{1}_{\mathcal{S}}$ and a certain $\kappa>0$. (This notion of the density of a set of primes is weaker than that of natural density.) Then Theorem 1.5 shows that $n / \sigma(n)$ has a continuous, strictly increasing distribution function when restricted to $\mathcal{S}$.

As a concrete example, we may take $\mathcal{S}$ to be the set of sums of two squares (where $\kappa=\frac{1}{2}$ ). We thus obtain another two-squares analogue of Davenport's result, this time with the elements of $\mathcal{S}$ counted without multiplicity.

Notation. If $F$ is a function on $[0,1]$, we write $\|F\|_{\infty}$ for the $L^{\infty}$-norm of $F$. We employ $O$ and $o$-notation, as well as the associated symbols $\ll$, $\gg$ and $\asymp$, with the usual meanings. All implied constants are absolute unless the dependence is explicitly indicated (for example, with a subscript). 


\section{Proof of Theorem 1.1}

We first show the existence of the limit (1.2) when the sharp cut-off condition $n / \sigma(n) \leq u$ is 'smoothed out'.

Lemma 2.1. Let $f$ be a multiplicative function satisfying the hypotheses of Theorem 1.1. For every continuous function $\psi$ on $[0,1]$, the limit

$$
\lim _{x \rightarrow \infty} \frac{1}{x} \sum_{n \leq x} f(n) \psi\left(\frac{n}{\sigma(n)}\right)
$$

exists.

Proof. Since $\psi$ is continuous on $[0,1]$, the Weierstrass approximation theorem allows us to choose a sequence of polynomials $p_{m}(x)$ with $\left\|\psi-p_{m}\right\|_{\infty} \leq 1 / m$. Since the arithmetic function $f(n)(n / \sigma(n))^{k}$ has a mean value for all nonnegative integers $k$, it follows that

$$
\mu_{m}:=\lim _{x \rightarrow \infty} \frac{1}{x} \sum_{n \leq x} f(n) p_{m}\left(\frac{n}{\sigma(n)}\right)
$$

exists for each $m$. In fact, the sequence $\left\{\mu_{m}\right\}$ is Cauchy. To see this, we start by observing that

$$
\left|\mu_{m}-\mu_{m^{\prime}}\right| \leq\left\|p_{m}-p_{m^{\prime}}\right\|_{\infty} \cdot \limsup _{x \rightarrow \infty} \frac{1}{x} \sum_{n \leq x}|f(n)| \leq \frac{2}{\min \left\{m, m^{\prime}\right\}} \cdot \limsup _{x \rightarrow \infty} \frac{1}{x} \sum_{n \leq x}|f(n)| .
$$

Since $f$ is bounded in mean square, Cauchy-Schwarz shows that

$$
\limsup _{x \rightarrow \infty} \frac{1}{x} \sum_{n \leq x}|f(n)| \leq \limsup _{x \rightarrow \infty}\left(\frac{1}{x} \sum_{n \leq x}|f(n)|^{2}\right)^{1 / 2} \ll_{f} 1 .
$$

Hence, $\left|\mu_{m}-\mu_{m^{\prime}}\right| \ll_{f} \min \left\{m, m^{\prime}\right\}^{-1}$, and so $\left\{\mu_{m}\right\}$ is a Cauchy sequence. Let $\mu=$ $\lim _{m \rightarrow \infty} \mu_{m}$. We claim that the limit in the statement of the lemma is precisely $\mu$. In fact, for every natural number $m$,

$$
\begin{aligned}
\limsup _{x \rightarrow \infty} & \left|\frac{1}{x} \sum_{n \leq x} f(n) \psi\left(\frac{n}{\sigma(n)}\right)-\mu\right| \\
& \leq\left|\mu-\mu_{m}\right|+\limsup _{x \rightarrow \infty}\left|\frac{1}{x} \sum_{n \leq x} f(n)\left(\psi\left(\frac{n}{\sigma(n)}\right)-p_{m}\left(\frac{n}{\sigma(n)}\right)\right)\right| \\
& \leq\left|\mu-\mu_{m}\right|+\left\|\psi-p_{m}\right\|_{\infty} \cdot \limsup _{x \rightarrow \infty} \frac{1}{x} \sum_{n \leq x}|f(n)| \ll_{f} \frac{1}{m},
\end{aligned}
$$

using (2.1) and (2.2) in the last step. Since $m$ can be taken arbitrarily large, it follows that $(1 / x) \sum_{n \leq x} f(n) \psi(n / \sigma(n)) \rightarrow \mu$, as desired. 
Proof of Theorem 1.1. We start by showing the existence of $D_{f}(u)$ for all $u \in[0,1]$, leaving the proof that $D_{f}(u)$ is continuous to the end. Since $D_{f}(1)$ is simply the mean value of $f$, we may assume that $0 \leq u<1$. Let $\psi$ be the characteristic function of $[0, u]$. Since $\psi$ is not continuous, we cannot directly apply Lemma 2.1. To work around this, we define, for positive integers $m$ large enough so that $u+1 / m<1$, functions

$$
\psi_{m}(x):= \begin{cases}1 & \text { if } 0 \leq x \leq u \\ 1-m(x-u) & \text { if } u<x<u+\frac{1}{m} \\ 0 & \text { if } u+\frac{1}{m} \leq x \leq 1\end{cases}
$$

Since each $\psi_{m}$ is continuous, Lemma 2.1 assures the existence of

$$
\mu_{m}=\lim _{x \rightarrow \infty} \sum_{n \leq x} f(n) \psi_{m}\left(\frac{n}{\sigma(n)}\right) .
$$

For $m^{\prime}>m$, we see that $\psi_{m^{\prime}}-\psi_{m}$ is supported on $[u, u+1 / m]$, and $\left\|\psi_{m}-\psi_{m^{\prime}}\right\|_{\infty} \leq 1$. Hence,

$$
\begin{aligned}
\left|\mu_{m}-\mu_{m^{\prime}}\right| & \leq \limsup _{x \rightarrow \infty} \frac{1}{x} \sum_{\substack{n \leq x \\
u \leq n / \sigma(n) \leq u+1 / m}}|f(n)| \\
& \ll_{f} \limsup _{x \rightarrow \infty}\left(\frac{1}{x} \sum_{\substack{n \leq x \\
u \leq n / \sigma(n) \leq u+1 / m}} 1\right)^{1 / 2}=\left(D\left(u+\frac{1}{m}\right)-D(u)\right)^{1 / 2} .
\end{aligned}
$$

Since $D$ is continuous, the final expression tends to 0 as $m$ tends to infinity. Thus, the sequence of $\mu_{m}$ is Cauchy with limit $\mu$, say. Notice that

$$
\begin{aligned}
\limsup _{x \rightarrow \infty} & \left|\frac{1}{x} \sum_{n \leq x} f(n) \psi\left(\frac{n}{\sigma(n)}\right)-\mu\right| \\
& \leq\left|\mu-\mu_{m}\right|+\limsup _{x \rightarrow \infty}\left|\frac{1}{x} \sum_{n \leq x} f(n)\left(\psi\left(\frac{n}{\sigma(n)}\right)-\psi_{m}\left(\frac{n}{\sigma(n)}\right)\right)\right| .
\end{aligned}
$$

Now $\psi-\psi_{m}$ is supported on $[u, u+1 / m]$, and $\left\|\psi-\psi_{m}\right\|_{\infty} \leq 1$; mimicking the process that led to (2.3), we see that the right-hand lim sup is $O_{f}\left((D(u+1 / m)-D(u))^{1 / 2}\right)$. From (2.3), we also have $\mu-\mu_{m} \ll_{f}(D(u+1 / m)-D(u))^{1 / 2}$. Since $m$ can be taken arbitrarily large, we conclude that the limit defining $D_{f}(u)$ exists and equals $\mu$.

The continuity of $D_{f}$ is now easy and in fact was essentially handled above. Indeed, a computation analogous to (2.3) shows that for every $u, v \in[0,1]$, we have $D_{f}(u)-D_{f}(v) \ll_{f}|D(u)-D(v)|^{1 / 2}$. Since $D$ is continuous on $[0,1]$, it follows that $D_{f}$ is continuous as well.

Corollary 1.2 will be deduced from the following two results. Proposition 2.2, which admits a completely elementary proof, is due essentially to Wintner (see, for 
example, [11, Corollary 2.3, pages 51-52]). Proposition 2.3, which lies substantially deeper, was first established by Delange [3] (compare with [11, Theorem 1.1, page 234]).

Proposition 2.2. Let $f$ be a multiplicative function satisfying (1.3). Then $f$ has a mean value. This mean value can be expressed explicitly as

$$
\prod_{p}\left(1-\frac{1}{p}\right)\left(1+\frac{f(p)}{p}+\frac{f\left(p^{2}\right)}{p^{2}}+\cdots\right) .
$$

Proposition 2.3. Let $f$ be a multiplicative function with $|f(n)| \leq 1$ for all $n \in \mathbb{N}$. If the series

$$
\sum_{p} \frac{1-f(p)}{p}
$$

converges, then $f$ has a mean value, again given by (2.4).

Proof of Corollary 1.2. Suppose first that $f$ is bounded in mean square and that (1.3) is satisfied. For each nonnegative integer $k$, let $f_{k}(n)=f(n)(n / \sigma(n))^{k}$. (Thus, $f=f_{0}$.) Since $\left|f_{k}\left(p^{j}\right)\right| \leq\left|f\left(p^{j}\right)\right|$, the double series in (1.3) remains convergent if $f$ is replaced by any of the $f_{k}$. Since $f_{k}(p)=f(p)+O_{k}(|f(p)| / p)$ and $\sum_{p}|f(p)-1| / p$ converges, to show that $\sum_{p}\left|f_{k}(p)-1\right| / p$ converges, it is enough to show that $\sum_{p}|f(p)| / p^{2}$ converges. But this is clear, since

$$
\sum_{p} \frac{|f(p)|}{p^{2}} \leq \sum_{p} \frac{|f(p)-1|}{p^{2}}+\sum_{p} \frac{1}{p^{2}}<\infty .
$$

So by Proposition 2.2, each $f_{k}$ possesses a mean value. This shows that the hypotheses of Theorem 1.1 hold for $f$.

Now let us assume instead that $|f(n)| \leq 1$ for all $n$ and that the series (1.4) converges. With $f_{k}$ defined as in the last paragraph, each $f_{k}$ is a multiplicative function taking values in the unit disc. Since $f_{k}(p)=f(p)+O_{k}(1 / p)$ and (1.4) converges, the series $\sum_{p}\left(1-f_{k}(p)\right) / p$ also converges. So by Proposition 2.3 , each $f_{k}$ has a mean value. Since $f$ is clearly bounded in mean square, the hypotheses of Theorem 1.1 are satisfied.

To prove Corollary 1.3, we make use of a celebrated theorem of Halász [6] (for other expositions, see [4, Ch. 6] or [11, Theorem 3.1, page 304]).

Proposition 2.4. Suppose that $f$ is a multiplicative function satisfying $|f(n)| \leq 1$ for all $n \in \mathbb{N}$. Then $f$ has mean value zero if and only if one of the following holds.

(i) There is a real number $\beta$ so that $f\left(2^{j}\right)=-2^{i j \beta}$ for each positive integer $j$. Moreover, the series

$$
\sum_{p} \frac{1-\operatorname{Re}\left(f(p) p^{-i \beta}\right)}{p}
$$

converges for this $\beta$.

(ii) The series (2.5) diverges for every real $\beta$. 
Proof of Corollary 1.3. This will be a corollary of the proof of Theorem 1.1, rather than the result itself. As above, let $f_{k}(n):=f(n)(n / \sigma(n))^{k}$. Since $f$ has mean value zero but there is no $\beta$ with $f\left(2^{j}\right)=-2^{i j \beta}$ for all $j$, it must be that (2.5) diverges for every real $\beta$. Since $f_{k}(p)=f(p)+O(1 / p)$, the series (2.5) remains divergent for every real $\beta$ if $f$ is replaced by any of the $f_{k}$. So by Proposition 2.4 again, each $f_{k}$ has mean value zero.

Referring back to the proof of Lemma 2.1, it follows that if $\psi$ is any continuous function on $[0,1]$, then $(1 / x) \sum_{n \leq x} f(n) \psi(n / \sigma(n)) \rightarrow 0$. Now referring to the proof of Theorem 1.1, we see that $D_{f}(u)$ vanishes identically, as desired.

\section{Proof of Theorem $\mathbf{1 . 5}$}

Let $f$ be a nonnegative multiplicative function satisfying the conditions of Theorem 1.5. For each real $x \geq 1$, we introduce the distribution function

$$
F_{x}(u):=\frac{1}{S(f ; x)} \sum_{\substack{n \leq x \\ \log (n / \sigma(n)) \leq u}} f(n) .
$$

The reason for working with $\log (n / \sigma(n))$ instead of directly with $n / \sigma(n)$ is to ensure that the characteristic function of $F_{x}$ is amenable to analysis; this will be important later. Theorem 1.5 is equivalent to the claim that the $F_{x}$ converge weakly to a continuous distribution function $F$ that is strictly increasing on $(-\infty, 0]$. Indeed, $\tilde{D}_{f}$ and $F$ are related by the change of variables $\tilde{D}_{f}\left(e^{u}\right)=F(u)$.

Our attack proceeds in three stages. First, we show the existence of the limiting distribution $F$. Next, we prove the continuity of $F$. Finally, we establish that $F$ is strictly increasing.

3.1. Existence. We will apply Lévy's convergence theorem, a well-known result drawn from the probabilist's toolchest (see, for example, [1, Corollary 1, page 350]).

Proposition 3.1. Suppose that $\left\{F_{x}\right\}$ is any collection of distribution functions indexed by real numbers $x \geq 1$. For each $x \geq 1$, let $\phi_{x}(t)$ be the characteristic function of $F_{x}$. The following two statements are equivalent.

(i) The $F_{x}$ converge weakly to a distribution function $F$, as $x \rightarrow \infty$.

(ii) As $x \rightarrow \infty$, the $\phi_{x}$ converge pointwise on all of $\mathbb{R}$ to a function $\psi$ that is continuous at 0 .

When (ii) holds, $\psi$ is the characteristic function of the limiting distribution $F$.

To evaluate the limit of the $\phi_{x}$ for our choice (3.1) of $\left\{F_{x}\right\}$, we need the following versatile theorem of Wirsing [12, Satz 1.1.1].

Proposition 3.2. Suppose that $f$ is a complex-valued multiplicative function with the property that as $x \rightarrow \infty$,

$$
\sum_{p \leq x} f(p) \frac{\log p}{p} \sim \kappa \log x
$$


for some real $\kappa>0$. Suppose also that $f(p)$ is bounded and that

$$
\sum_{p} \sum_{j \geq 2} \frac{\left|f\left(p^{j}\right)\right|}{p^{j}}<\infty
$$

If $\kappa \leq 1$, suppose further that

$$
\sum_{p^{j} \leq x}\left|f\left(p^{j}\right)\right| \ll_{f} \frac{x}{\log x} \quad(\text { for } x \geq 2) .
$$

Finally, suppose that

$$
\sum_{p} \frac{1}{p}(|f(p)|-\operatorname{Re}(f(p)))<\infty .
$$

Then as $x \rightarrow \infty$,

$$
\sum_{n \leq x} f(n) \sim \frac{e^{-\gamma \kappa}}{\Gamma(\kappa)} \frac{x}{\log x} \prod_{p \leq x}\left(1+\frac{f(p)}{p}+\frac{f\left(p^{2}\right)}{p^{2}}+\cdots\right) .
$$

Here $\gamma$ is the Euler-Mascheroni constant, and $\Gamma(\cdot)$ is the classical Gamma-function.

Proof of the EXISTENCE OF THE Limiting Distribution $F$. The characteristic function $\phi_{x}$ of $F_{x}$ is given by

$$
\phi_{x}(t)=\frac{1}{S(f ; x)} \sum_{n \leq x} f(n)\left(\frac{n}{\sigma(n)}\right)^{i t} .
$$

Because of the conditions on $f$ in Theorem 1.5, Proposition 3.2 yields an asymptotic formula for $S(f ; x)$. Proposition 3.2 may also be applied to give an analogous formula for the partial sums of $f(n)(n / \sigma(n))^{i t}$. To see this, notice that $\left|f(n)(n / \sigma(n))^{i t}\right|=f(n)$, and that

$$
\left(\frac{p}{\sigma(p)}\right)^{i t}-1=\left|\exp \left(i t \log \frac{p}{p+1}\right)-1\right| \leq\left|t \log \frac{p}{p+1}\right|=|t| \log \frac{p+1}{p} \leq \frac{|t|}{p},
$$

so that

$$
f(p)\left(\frac{p}{\sigma(p)}\right)^{i t}=f(p)+O\left(\frac{|t|}{p}\right) .
$$

The hypotheses of Proposition 3.2, with the same $\kappa$ as in (1.5), are now easily seen to follow from the conditions assumed on $f$. Comparing the asymptotic estimates obtained from (3.2) for $f(n)$ and $f(n)(n / \sigma(n))^{i t}$, we find that as $x \rightarrow \infty$ with $t$ fixed,

$$
\phi_{x}(t) \sim \prod_{p \leq x}\left(\left(\sum_{j=0}^{\infty} \frac{f\left(p^{j}\right)}{p^{j}}\left(\frac{p^{j}}{\sigma\left(p^{j}\right)}\right)^{i t}\right) \cdot\left(\sum_{j=0}^{\infty} \frac{f\left(p^{j}\right)}{p^{j}}\right)^{-1}\right) .
$$

For notational convenience, let us write

$$
\alpha_{p}(t)=\sum_{j=0}^{\infty} \frac{f\left(p^{j}\right)}{p^{j}}\left(\frac{p^{j}}{\sigma\left(p^{j}\right)}\right)^{i t} \quad \text { and } \quad \Delta_{p}=\sum_{j=0}^{\infty} \frac{f\left(p^{j}\right)}{p^{j}} .
$$


Note that $\Delta_{p}$ is finite for every $p$, by (1.6). Since the terms in the series defining $\alpha_{p}(t)$ are bounded in absolute value by the corresponding terms in $\Delta_{p}$, the series for $\alpha_{p}(t)$ converges uniformly, and so $\alpha_{p}(t)$ is continuous everywhere. Let

$$
\eta_{p}=\sum_{j=2}^{\infty} \frac{f\left(p^{j}\right)}{p^{j}}
$$

We will show below that for all primes $p$ exceeding a certain constant $p_{0}$,

$$
\alpha_{p}(t) \Delta_{p}^{-1}=1+O\left(\frac{1+|t|}{p^{2}}+\eta_{p}\right)
$$

we allow both $p_{0}$ and the implied constant to depend on $f$. Now $\sum_{p} 1 / p^{2}<\infty$, and (1.6) asserts that $\sum_{p} \eta_{p}<\infty$. Assuming for the time being that (3.5) has been established, we see that the series $\sum_{p>p_{0}}\left|\alpha_{p}(t) \Delta_{p}^{-1}-1\right|$ converges uniformly on any interval $[-T, T]$. Consequently, the infinite product

$$
\prod_{p>p_{0}} \alpha_{p}(t) \Delta_{p}^{-1}
$$

converges to a function of $t$ that is continuous everywhere. Of course, the finite product $\prod_{p \leq p_{0}} \alpha_{p}(t) \Delta_{p}^{-1}$ is also continuous on all of $\mathbb{R}$. We conclude from (3.4) that as $x \rightarrow \infty$,

$$
\phi_{x}(t) \rightarrow \psi(t),
$$

where

$$
\psi(t):=\prod_{p}\left(\left(\sum_{j=0}^{\infty} \frac{f\left(p^{j}\right)}{p^{j}}\left(\frac{p^{j}}{\sigma\left(p^{j}\right)}\right)^{i t}\right) \cdot\left(\sum_{j=0}^{\infty} \frac{f\left(p^{j}\right)}{p^{j}}\right)^{-1}\right)
$$

is continuous everywhere. So by Lévy's criterion, the $F_{x}$ converge weakly to a limiting distribution $F$ with characteristic function $\psi$.

It remains to establish the estimate (3.5). Using (3.3) once more,

$$
\begin{aligned}
\alpha_{p}(t) \Delta_{p}^{-1} & =\left(1+\frac{f(p)}{p}\left(\frac{p}{\sigma(p)}\right)^{i t}+O\left(\eta_{p}\right)\right) \Delta_{p}^{-1} \\
& =\left(1+\frac{f(p)}{p}+O\left(\frac{|t|}{p^{2}}\right)\right) \Delta_{p}^{-1}+O\left(\eta_{p}\right) .
\end{aligned}
$$

Now $\Delta_{p}=1+f(p) / p+\eta_{p}$. We are assuming that $f(p)=O(1)$ and that $\sum_{p} \eta_{p}$ converges; thus, we can choose $p_{0}$ so that $0 \leq \Delta_{p}-1 \leq 1 / 2$ for all $p>p_{0}$. Since $1 /(1+z)=1-z+O\left(z^{2}\right)$ for $|z| \leq 1 / 2$, we have for $p>p_{0}$ that

$$
\begin{aligned}
\Delta_{p}^{-1} & =1-\left(\Delta_{p}-1\right)+O\left(\left(\Delta_{p}-1\right)^{2}\right) \\
& =1-\frac{f(p)}{p}+O\left(\eta_{p}+\frac{1}{p^{2}}\right) .
\end{aligned}
$$

Substituting (3.8) into (3.7) yields (3.5). 
3.2. Continuity. Let $X_{p}$ denote the discrete random variable taking the value $\log \left(p^{j} / \sigma\left(p^{j}\right)\right)$ with probability $\left(1 / \Delta_{p}\right) f\left(p^{j}\right) / p^{j}$, for each $j=0,1,2, \ldots$ Let $\phi_{X_{p}}$ be the characteristic function of $X_{p}$. Then

$$
\begin{aligned}
\phi_{X_{p}}(t)=\mathrm{E}\left[e^{i t X_{p}}\right] & =\sum_{j=0}^{\infty} e^{i t \log p^{j} / \sigma\left(p^{j}\right)} \cdot \operatorname{Prob}\left(X_{p}=\log \frac{p^{j}}{\sigma\left(p^{j}\right)}\right) \\
& =\left(\sum_{j=0}^{\infty} \frac{f\left(p^{j}\right)}{p^{j}}\left(\frac{p^{j}}{\sigma\left(p^{j}\right)}\right)^{i t}\right)\left(\sum_{j=0}^{\infty} \frac{f\left(p^{j}\right)}{p^{j}}\right)^{-1},
\end{aligned}
$$

which is precisely the $p$ th term in the product formula (3.6). This shows (see [5, Equation (12)]) that $\psi(t)$ is the infinite convolution of the $\phi_{X_{p}}$, as $p$ ranges over the primes. The following result of Lévy [4, Lemma 1.22, page 46] provides the approach that we will adopt in our proof that $\psi(t)$ is continuous.

Lemma 3.3. Suppose that $\psi$ is an infinite convergent convolution of purely discontinuous distribution functions $\phi_{1}, \phi_{2}, \ldots$; that is, $\psi=\phi_{1} * \phi_{2} * \cdots$. Let $d_{k}$ be the maximal jump of each $\phi_{k}$. If $\sum_{k=1}^{\infty}\left(1-d_{k}\right)$ diverges, then the limit distribution is continuous.

Proof of Continuity. Let $d_{p}$ be the maximal jump in the distribution function of $X_{p}$. By Lemma 3.3, it suffices to show that $\sum_{p}\left(1-d_{p}\right)$ diverges. Now the distribution function of $X_{p}$ has jumps of size $\left(1 / \Delta_{p}\right) f\left(p^{j}\right) / p^{j}$ at the points $\log \left(p^{j} / \sigma\left(p^{j}\right)\right)$, where $j$ ranges over those nonnegative integers with $f\left(p^{j}\right) \neq 0$. Taking $j=0$, we see that there is a jump at $x=0$ of size $1 / \Delta_{p}$. Since $f(p) / p$ and $\eta_{p}$ both tend to zero, we may choose $p_{0}$ so that $\Delta_{p}=1+f(p) / p+\eta_{p}<2$ for all $p>p_{0}$. For these values of $p$, we have $1 / \Delta_{p}>1 / 2$, and so the largest jump must occur at $x=0$. Hence, $d_{p}=1 / \Delta_{p}$ for $p>p_{0}$, and

$$
\sum_{p}\left(1-d_{p}\right) \geq \sum_{p>p_{0}} \frac{\Delta_{p}-1}{\Delta_{p}} \geq \frac{1}{2} \sum_{p>p_{0}}\left(\Delta_{p}-1\right) \geq \frac{1}{2} \sum_{p>p_{0}} \frac{f(p)}{p} .
$$

Recall that $\sum_{p \leq x}(f(p) / p) \log p \sim \kappa \log x$ for a certain $\kappa>0$. By partial summation,

$$
\sum_{p \leq x} \frac{f(p)}{p} \sim \kappa \log \log x \quad(\text { as } x \rightarrow \infty) .
$$

Consequently, $\sum_{p}\left(1-d_{p}\right)$ diverges.

3.3. Strict monotonicity. Since we have already established the existence and continuity of $F$, we know at this point that $\tilde{D}_{f}$ is a well-defined, continuous function on $[0,1]$. Rather than prove that $F$ is strictly increasing on $(-\infty, 0]$, we prove directly that $\tilde{D}_{f}$ is strictly increasing on $[0,1]$. 
Proof that $\tilde{D}_{f}$ Is Strictly increasing. It suffices to show that for $u, v \in[0,1]$ with $v<u$,

$$
\liminf _{x \rightarrow \infty} \frac{1}{S(f ; x)} \sum_{\substack{n \leq x \\ v<n / \sigma(n) \leq u}} f(n)>0 .
$$

In proving (3.10), there is no loss of generality in assuming that $f$ is supported on squarefree integers. This is because $S(f ; x)$ and $S\left(f \mu^{2} ; x\right)$ have the same order of magnitude. To see this last claim, note that comparing the corresponding versions of (3.2) shows that

$$
S(f ; x) \sim S\left(f \mu^{2} ; x\right) \cdot \prod_{p \leq x}\left(1+\eta_{p}\left(1+\frac{f(p)}{p}\right)^{-1}\right),
$$

as $x \rightarrow \infty$. Since $\sum_{p} \eta_{p}<\infty$, the right-hand product converges as $x \rightarrow \infty$. Thus, $S(f ; x) \asymp S\left(f \mu^{2} ; x\right)$ for large $x$, as claimed.

Since $f(p)$ is bounded, (3.9) implies that the sum of the reciprocals of those $p$ with $f(p) \neq 0$ diverges. Since $|\log (p / \sigma(p))| \asymp 1 / p$, we may use the greedy algorithm to select a squarefree natural number $m$ with $f(m)>0$ and with $v<m / \sigma(m) \leq u$. We keep this $m$ fixed for the remainder of the argument. We let $y$ be a real parameter, viewed as fixed but eventually to be chosen very large. For now, we assume that $y$ exceeds the largest prime factor of $m$.

Consider the contribution to the sum in (3.10) from those $n=m q$ where $q$ is squarefree and coprime to $\Pi_{y}:=\prod_{p \leq y} p$. We will show that if $y$ is chosen sufficiently large, then this contribution is already enough to imply (3.10). Notice that

$$
\begin{aligned}
\sum_{\substack{q \leq x / m \\
\operatorname{gcd}\left(q, \Pi_{y}\right)=1 \\
v<m q / \sigma(m q) \leq u}} f(m q) & =f(m) \sum_{\begin{array}{c}
q \leq x / m \\
\operatorname{gcd}\left(q, \Pi_{y}\right)=1 \\
q / \sigma(q)>v \sigma(m) / m
\end{array}} f(q) \\
& \geq f(m) \sum_{\substack{q \leq x / m \\
\operatorname{gcd}\left(q, \Pi_{y}\right)=1}} f(q)\left(1-v \frac{\sigma(m)}{m} \cdot \frac{\sigma(q)}{q}\right) .
\end{aligned}
$$

Let $\mathbf{1}_{y}$ be the indicator function of those numbers coprime to $\Pi_{y}$. Set $a_{y}(n)=f(n) \mathbf{1}_{y}(n)$ and $b_{y}(n)=f(n)(\sigma(n) / n) \mathbf{1}_{y}(n)$. The sum in (3.11) can be written as

$$
S\left(a_{y} ; \frac{x}{m}\right)-v \frac{\sigma(m)}{m} S\left(b_{y} ; \frac{x}{m}\right) .
$$

By Proposition 3.2 and our assumption that $f$ is supported on squarefrees,

$$
S(f ; x) \sim \frac{e^{-\gamma \kappa}}{\Gamma(\kappa)} \frac{x}{\log x} \prod_{p \leq x}\left(1+\frac{f(p)}{p}\right) .
$$


Since $f(p)$ is bounded, the asymptotic relation (3.13) remains valid even if the product is shortened to be over the primes $p \leq x / m$. Now applying Proposition 3.2 to $a_{y}$,

$$
\begin{aligned}
S\left(a_{y} ; \frac{x}{m}\right) & \sim \frac{e^{-\gamma \kappa}}{\Gamma(\kappa)} \frac{x}{m \log x} \prod_{y<p \leq x / m}\left(1+\frac{f(p)}{p}\right) \\
& \sim \frac{1}{m} S(f ; x) \cdot \prod_{p \leq y}\left(1+\frac{f(p)}{p}\right)^{-1} .
\end{aligned}
$$

Similarly,

$$
S\left(b_{y} ; \frac{x}{m}\right) \sim \frac{1}{m} S(f ; x) \cdot \prod_{p \leq y}\left(1+\frac{f(p)}{p}\right)^{-1} \prod_{y<p \leq x / m} \frac{1+\frac{f(p)}{p}\left(1+\frac{1}{p}\right)}{1+\frac{f(p)}{p}} .
$$

Combining (3.11), (3.12), (3.14) and (3.15), we see that the lim inf in (3.10) is bounded below by

$$
\begin{aligned}
f(m) & \cdot \liminf _{x \rightarrow \infty}\left(\frac{S\left(a_{y} ; \frac{x}{m}\right)}{S(f ; x)}-v \frac{\sigma(m)}{m} \frac{S\left(b_{y} ; \frac{x}{m}\right)}{S(f ; x)}\right) \\
& =\frac{f(m)}{m}\left(\prod_{p \leq y}\left(1+\frac{f(p)}{p}\right)^{-1}\right)\left(1-v \frac{\sigma(m)}{m} \prod_{p>y} \frac{1+\frac{f(p)}{p}\left(1+\frac{1}{p}\right)}{1+\frac{f(p)}{p}}\right) .
\end{aligned}
$$

In the product over $p>y$, each term is at least 1 but at most $1+f(p) / p^{2} \leq 1+O\left(1 / p^{2}\right)$. Thus, that product tends to 1 as $y \rightarrow \infty$. It follows that if we fix $y$ to be sufficiently large, then (3.16) is positive. This completes the proof.

\section{References}

[1] P. Billingsley, Probability and Measure, 3rd edn. Wiley Series in Probability and Mathematical Statistics (John Wiley \& Sons, New York, 1995).

[2] H. Davenport, 'Über numeri abundantes', S.-Ber. Preuß. Akad. Wiss., math.-nat. Kl. (1933), 830-837.

[3] H. Delange, 'Sur les fonctions arithmétiques multiplicatives', Ann. Sci. Éc. Norm. Supér. (3) 78 (1961), 273-304.

[4] P. D. T. A. Elliott, Probabilistic number theory I: mean-value theorems, Grundlehren der Mathematischen Wissenschaften, 239 (Springer, New York-Berlin, 1979).

[5] P. Erdős and A. Wintner, 'Additive arithmetical functions and statistical independence', Amer. J. Math. 61 (1939), 713-721.

[6] G. Halász, 'Über die Mittelwerte multiplikativer zahlentheoretischer Funktionen', Acta Math. Acad. Sci. Hungar. 19 (1968), 365-403.

[7] G. H. Hardy and E. M. Wright, An Introduction to the Theory of Numbers, 6th edn. (Oxford University Press, Oxford, 2008).

[8] M. Kobayashi, On the Density of Abundant Numbers. PhD Thesis, Dartmouth College. 2010.

[9] P. Moree, 'Counting numbers in multiplicative sets: Landau versus Ramanujan', Math. Newsl. 21 (2011), 73-81.

[10] S. S. Pillai, 'Generalisation of a theorem of Mangoldt', Proc. Indian Acad. Sci., Sect. A. 11 (1940), 13-20. 
[11] W. Schwarz and J. Spilker, Arithmetical Functions, London Mathematical Society Lecture Note Series, 184 (Cambridge University Press, Cambridge, 1994).

[12] E. Wirsing, 'Das asymptotische Verhalten von Summen über multiplikative Funktionen. II', Acta Math. Acad. Sci. Hungar. 18 (1967), 411-467.

EMILY JENNINGS, Department of Mathematics, University of Georgia, Athens, Georgia 30602, USA

e-mail: emily@math.uga.edu

PAUL POLLACK, Department of Mathematics, University of Georgia,

Athens, Georgia 30602, USA

e-mail:pollack@uga.edu

LOLA THOMPSON, Department of Mathematics, University of Georgia, Athens, Georgia 30602, USA

e-mail: lola@math.uga.edu 